\title{
An Architecture for Software Defined Wireless Networking
}

\author{
Carlos J. Bernardos, Antonio de la Oliva, \\ Pablo Serrano, Albert Banchs \\ Universidad Carlos III de Madrid \\ Avda. Universidad, 30 \\ Leganes, Spain \\ \{cjbc, aoliva, pablo, banchs\}@it.uc3m.es
}

\author{
Luis M. Contreras \\ Telefónica I+D \\ c/ Don Ramón de la Cruz, 82-84 \\ Madrid, Spain \\ lmcm@tid.es
}

\author{
Hao Jin \\ InterDigital Communications, Inc \\ 781 Third Avenue, King of Prussia \\ Pennsylvania, USA \\ hao.jin@interdigital.com
}

\author{
Juan Carlos Zúñiga \\ InterDigital Communications, LLC \\ 1000 Sherbrooke Street West \\ Montreal, Canada \\ juancarlos.zuniga@interdigital.com
}

\begin{abstract}
Software Defined Networking (SDN), characterized by a clear separation of the control and data planes, is being adopted as a novel paradigm for wired networking. With SDN, network operators can run their infrastructure more efficiently, supporting a faster deployment of new services while enabling key features such as virtualization. In this article, we adopt an SDN-like approach applied to wireless mobile networks that will not only benefit from the same features as in the wired case, but also will leverage on the distinct features of mobile deployments to push improvements even further. We illustrate with a number of representative use cases the benefits from the adoption of the proposed architecture, which is detailed in terms of modules, interfaces and high-level signaling. We also review the ongoing standardization efforts, and discuss the potential advantages, weaknesses and the need for a coordinated approach.
\end{abstract}

Keywords - SDN, Wireless, Standardization, ONF, OpenFlow, OmniRAN, Access, Backhaul

\section{INTRODUCTION}

The telecommunications sector is experiencing a major revolution that will shape the way networks and services are designed and deployed for the next decade. We are witnessing an explosion in the number of applications and services demanded by users, which are now really capable of accessing them on the move. In order to cope with such a demand, some network operators are now following a cloud computing paradigm, enabling the reduction of the overall costs by outsourcing communication services from specific hardware in the operators' core to server farms scattered in datacenters. These services have different characteristics if compared with conventional IT services that have to be taken into account in this cloudification process [1]

Virtualization of functions also provides operators with tools to deploy new services much faster, as compared to the traditional use of monolithic and tightly integrated dedicated machinery [2]. As a natural next step, mobile network operators need to re-think how to evolve their existing network infrastructures and how to deploy new ones to address the challenges posed by the increasing customers' demands, as well as by the huge competition among operators. All these changes are triggering the need for a modification in the way operators and infrastructure providers operate their networks, as they need to significantly reduce the costs incurred in deploying a new service and operating it.

Some of the mechanisms that are being considered and already adopted by operators include: sharing of network infrastructure to reduce costs, virtualization of core servers running in data centers as a way of supporting their loadaware elastic dimensioning, and dynamic energy policies to reduce the monthly electricity bill. However, this has proved to be tough to put in practice, and not enough. Indeed, it is not easy to deploy new mechanisms in a running operational network due to the high dependency on proprietary (and sometime obscure) protocols and interfaces, which are complex to manage and often require configuring multiple devices in a decentralized way.

Building on the revolutionary forward thinking in computer networking, software defined networking (SDN) is currently being considered as an alternative to classic distributed approaches based on highly specialized hardware executing standardized protocols. Up to now, most of the key use cases used to present the benefits of the SDN paradigm have been limited to wired environments (e.g., Google uses SDN in its datacenters [3]).

In this article we analyze the potential of applying the SDN paradigm to mobile wireless networks. First, we identify use cases where a wireless SDN approach could bring additional benefits. Then, we derive the main characteristics of a wireless SDN mobile operator's architecture, paying special attention to the main functions and interfaces. In order to illustrate the 
operation of the proposed mobile wireless SDN framework, we introduce the high-level interactions required between the defined functions to support an example use case of interest for operators. We finish this article with a review of current standardization efforts and trends in this arena, and we then elaborate on the need for specific actions towards the standardization of what we call Software Defined Wireless Networking (SDWN).

\section{BACKGROUND: SDN, OPENFLOW, CAPWAP AND RE- CONFIGURABLE WIRELESS DEVICES}

Software Defined Networking is a networking paradigm [4] that separates the control and the data forwarding planes. Such separation allows for quicker provisioning and configuration of network connections. With SDN, network administrators can program the behavior of both the traffic and the network in a centralized way, without requiring independently accessing and configuring each of the network's hardware devices. This approach decouples the system that makes decisions about where traffic is sent (i.e., control plane) from the underlying system that forwards traffic to the selected destination (i.e., data plane). Among other advantages, this simplifies networking as well as the deployment of new protocols and applications. In addition, by enabling programmability on the traffic and the devices, an SDN network might be much more flexible and efficient than a traditional one.

Figure 1 shows a logical view of the commonly accepted SDN reference architecture [4]. In this architecture, the intelligence is centralized in software-based SDN controllers, which have a global view of the network and are capable of controlling, in a vendor-independent way, the network devices. These network devices are no longer required to implement and understand many different network protocols standards; instead, they can provide such functionality by accepting instructions from SDN controllers. This saves a lot of time and resources, as the network behavior can be easily controlled by programming it in the centralized controllers, rather than using custom configurations in many different devices scattered across the network.

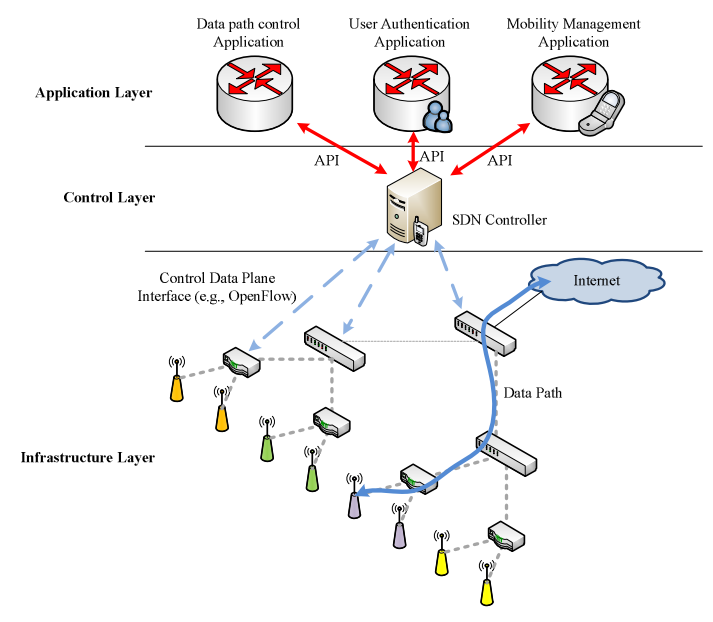

Figure 1. SDN reference architecture
A key requirement to deploy an SDN architecture, such as the one defined above, is to standardize the interface to control the mobile devices. This can be done with OpenFlow [5], which is a standardized interface between the control and forwarding layers of the SDN architecture. The vendor-agnostic nature of OpenFlow facilitates the integration of heterogeneous devices in a common way, simplifying the operation of multi-vendor infrastructures, which are typically found in commercial telecom networks. It allows accessing and modifying the forwarding plane of network devices such as switches and routers by the definition of specific rules for matching packet flows against a selection of layer- 2 to layer-4 packet header's field values and the flows' ingress port number. These rules take the form of entries in forwarding flow tables residing in the network devices.

It should be noted that this separation of control and data plane for the switching fabric also exists, to some extent, in the wireless domain. Indeed, the IETF standardized several years ago the Control And Provisioning of Wireless Access Points (CAPWAP) protocol [6], which centralizes the control in wireless networks. In principle, CAPWAP is technology agnostic and requires specific bindings for each considered access standard, although so far only the binding for 802.11 has been defined. Radio configuration is expressed in terms of management information base elements included in the standard, such as the operating channel or the transmission power, but also the beacon interval or the contention parameters used by the medium access scheme. With CAPWAP, control frames are delivered to a central controller, which is responsible for MAC layer control, in a way that can be easily related to the way OpenFlow delivers to the controller information about new incoming flows.

Along the same lines, but in a more visionary approach, a novel paradigm for the re-programming of wireless interfaces has been proposed in [7]. In this vision, wireless nodes execute a Wireless MAC Processor, in charge of running "MAClets", i.e., programs specifying the MAC protocol. In this way, the central controller can dynamically upload the protocol to use at a given point in time, e.g., changing from CSMA/CA to TDMA-based access when the traffic load increases.

There have also been some efforts looking at the use of SDN in mobile networks, such as MobileFlow [8], which proposes an SDN approach for the core network. However, the proposed solution does not provide an integrated vision including the wireless access.

While efforts in SDN so far have mostly focused on wired and core networks, we believe that the adoption of a similar concept for wireless access and backhaul environments can be even more beneficial. Indeed, the control plane of wireless networks is more complex than the one of wired networks, and therefore higher gains can be achieved from the increased flexibility provided by an SDN approach.

\section{USE CASES}

Before addressing the design of an SDWN architecture, in this section we describe some use cases in which the adoption of a 
software defined wireless networking approach brings significant advantages. Rather than identifying an exhaustive set of use cases, the purpose of these use cases is simply to illustrate some of the potential advantages of adopting an SDN approach in a mobile wireless environment.

\section{A. Virtualization}

Current deployments support virtualization to some extent, but existing network devices and mechanisms are not designed to support dynamic re-configuration required for timely and efficient sharing of resources. More specifically, although servers can be virtualized and allocated to different physical resources in almost real time, the paths communicating these virtual appliances with the rest of the world still require manual installation, or interaction with protocols which are not designed for dynamic and fast responses. Hence, current adaptation mechanisms in network technologies are seen as one of the bottlenecks for the general deployment of virtualized infrastructures. Previous approaches for core network virtualization (e.g., PlanetLab ${ }^{1}$ or $\mathrm{GENI}^{2}$ ) have implemented different overlay networks so researchers can run their experiments by time-sharing access to resources. However, these approaches operate in a coarse time scale, need manual planning and dimensioning, and lack the required timeliness for their operation in production networks. These approaches differ from actual trends in virtualization for the access network, more focused on sharing and enforcing the radio and transport network resources among different operators.

Following this trend, the adoption of SDN (with relatively mature technologies such as, e.g., OpenFlow or ForCES ${ }^{3}$ ) should improve support for timely and efficient virtualization of a wireless network, but there are some challenges that need to be successfully tackled. First, in order to provide the required flexibility in terms of network topology and architecture demanded by virtualization applications, an SDN network must be able to implement a wide set of control logics that are simultaneously applied to the same set of physical resources. The support of several different control logics on the same network raises scalability and compatibility issues. For example, each of the control logics may be working on top of a different realization of the network, each of them requiring fast reaction upon changes in the underlying physical infrastructure. This challenge calls for a scalable network orchestration mechanism, which coordinates SDN control and data plane operations and resolves any contentions between different control logics.

In addition, the different control logics must be able to work in an isolated way. This must be implemented in two different planes. On the one hand, traffic from a certain instantiation of a virtual operator must be isolated from traffic belonging to a different virtual operator, for security and privacy reasons. On the other hand, changes performed in the virtual infrastructure of an operator must be isolated from the rest of virtual

\footnotetext{
${ }^{1}$ https://www.planet-lab.org/

${ }^{2} \mathrm{http}: / /$ www.geni.net/

${ }^{3} \mathrm{http}$ //datatracker.ietf.org/wg/forces/charter/
}

instances sharing the network, e.g., a change in the configuration of the virtual infrastructure must not affect the rest of the instances running on top of the real deployment.

Another key issue is the allocation and sharing of network resources, considering both time resolution and isolation. This not only prevents resource wastage due to the coordination and sharing, but also allows gaining from statistical multiplexing, so the use of this solution is substantially more efficient than having independent deployments.

Note that SDN is independent from and complementary to another notable virtualization initiative, the Network Functions Virtualisation (NFV) [2]. While SDN focuses on the virtualization of network devices, NFV aims to enable the virtualization of network services and functions, such as NAT, firewall and cellular core functions, so that the time to deploy services can be shortened and operator CAPEX/OPEX can be reduced. One example of the mutual-benefit between NFV and SDN is that, on the one hand, NFV may improve the efficiency and flexibility of SDN's control plane services; and on the other hand, SDN may ensure the delivery and quality of the network traffic between NFV's virtualized functions.

\section{B. QoE-aware network operation}

Current networks are provisioned and operated towards providing a certain level of Quality of Service (QoS). However, this does not always ensure a minimum "Quality of Experience" (QoE) to the user. The QoE of a service is determined by roughly three factors: the service architecture (e.g., server capabilities, caches, and their location), the core network performance, and the service provided at the "wireless last mile", i.e., the combination of the wireless link(s) - including the backhaul - and the capabilities of the terminal. With current architectures, a service provider has to anticipate its needs in terms of infrastructure, negotiate an SLA based on these estimations, and at most try to adapt to users' experiences, in a coarse time-scale, e.g., by changing the encoding of the video being served (as YouTube does). It is clear that such a scenario precludes an efficient use of resources, as the service provider has no mechanism to react in a timely manner to the changing conditions, because the service provider has limited indicators of the user's performance in real time, nor the ability to quickly deploy more architectural elements or improve the SLA with the network providers.

Furthermore, mobile networks intrinsically present a need for integrating QoS objectives in the radio part (i.e., service layer) and the backhaul network (i.e., transport layer) [9]. This drives the necessity of dynamically orchestrating resources in both layers for providing a uniform and efficient QoE.

The use of a software defined wireless networking architecture would allow the network to offer to the service provider an API to control how the networks behave to serve the traffic that matches a certain set of rules (of course, the degree of control would depend on the agreements between network operators and service providers and the kind of requested control). Furthermore, through this API the provider will be also able to dynamically change the forwarding paths of the flows (in both directions), so traffic transverses 
opportunistically deployed middleware, which can for instance serve as data caches, or video transcoders. Finally, the provider, now acting as a true service composer, can use the API to change the behavior of the "wireless last mile" in three ways: first, by dynamically prioritizing traffic at the last hop, so in case of poor wireless conditions, some packets (e.g., I frames of a video stream) are provided with a better service than others (e.g., B frames), because are marked as more important; second, by being aware of the service experienced by the user, thus timely adapting following his/her preferences; third, by supporting traffic on- and off-loading, based on these preferences and the availability of different communication links (each with a different performance vs. cost trade-off). In this way, the provider personalizes the operation of the network after user's behavior and preferences.

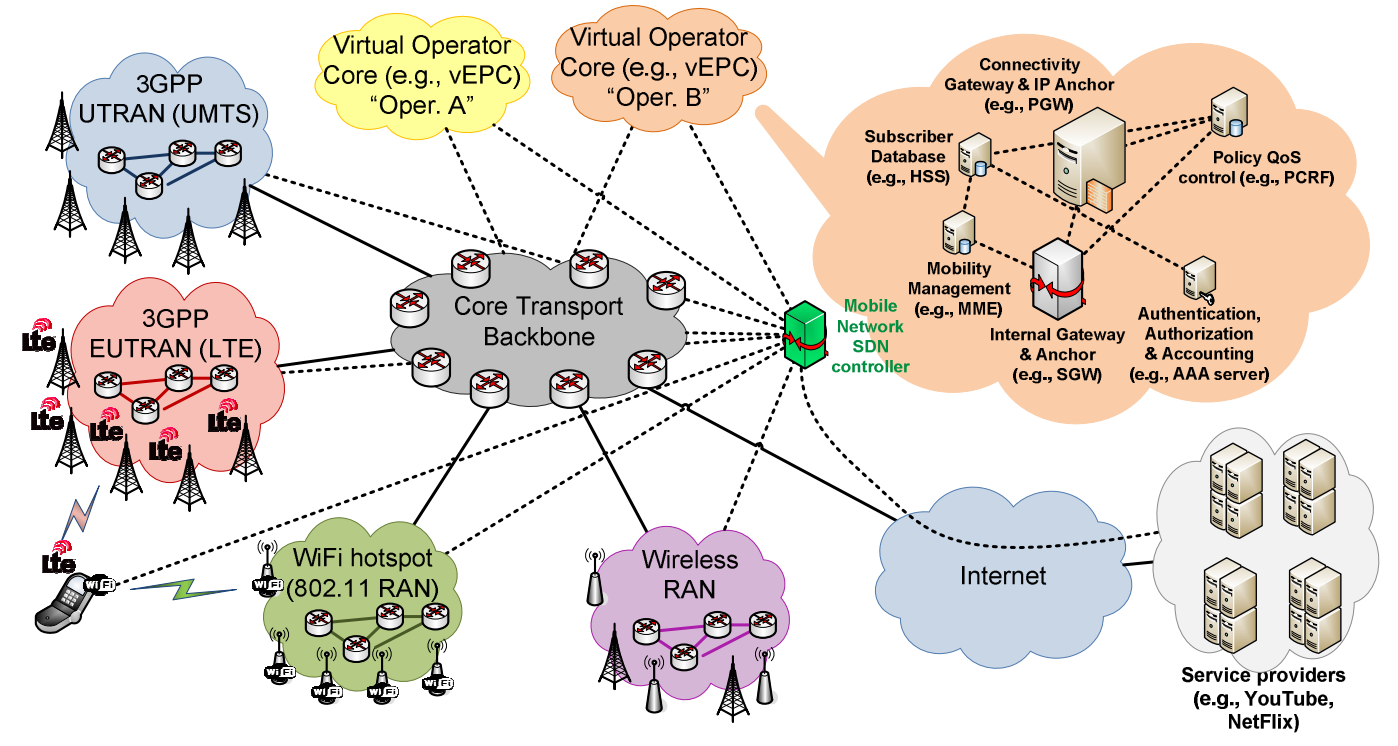

Figure 2. SDN-based mobile network architecture

The above requires the design of customer-oriented traffic management services, which are able to connect different applications to different technologies and to adapt content to network conditions and available resources. By considering the specifics of each application, a differentiated service can be provided to different flows. For example, quality and/or timely delivery of a given flow can be balanced against for extra capacity for a different flow with more stringent delay requirements. This requires the ability to design and coordinate offloading/seamless mobility mechanisms across the heterogeneous (technology and operator) networks.

\section{Network access selection and mobility control}

Existing mobile terminals are generally equipped with multiple network interfaces, typically $\mathrm{WiFi}$ and cellular. This, together with the proliferation of femtocells, WiFi hot-spots, and WiFi access to fixed residential home gateways, has complicated the process of selecting the best access technology at each moment. A mobile node may decide to use the available attachment options sequentially (i.e., move all traffic from one technology to another one) or simultaneously (i.e., move selected flows from one access to another [10]). Despite that telecom operators can offer both residential fixed and mobile services, WiFi hot-spot accesses are usually not directly managed by the operator and their characteristics make it challenging to ensure a given QoS (as opposed to cellular accesses). Because of these factors, the decision of how to select and opportunistically use the heterogeneous available accesses is not a trivial one.

Additionally, the network might want to keep control on how the traffic (on an application granularity level) is delivered to the mobile terminal. This involves, for example, selective and opportunistic traffic offloading. By using an SDWN solution, an API could be offered to external parties (such as service providers), so they can influence the decision of which access technology is used to deliver a certain type of traffic to a specific mobile terminal or group of users. This particular scenario could also benefit from enabling the programmability of the mobile node as well, for example to enable easy control from the network side on how the different available network accesses are used by the traffic generated by applications running on the mobile.

\section{SDWN ARCHITECTURE}

We next describe a generic software defined wireless networking (SDWN) architecture. Such an architecture aims at bringing the benefits of logical orchestration: by providing well-defined interfaces for control plane functions and enabling a richer flexibility in user plane traffic handling.

\section{A. System View}

Figure 2 shows an SDWN-based architecture of a mobile network operator, where a solid line in the figure denotes a user plane connection, and a dashed one is used for the control plane. We take the 3GPP Evolved Packet System as reference 
architecture to link the proposed concepts with a well established and understood system architecture.

A mobile network typically exhibits multiple heterogeneous Radio Access Networks (RANs) connected to a common transport core network. Note the connection between the last network entity providing radio access and the core transport network might involve a wired or wireless backhaul network (shown as part of the RAN in the figure) by using a combination of technologies (e.g., fiber optic, microwave) and topologies (e.g., ring structure, daisy chain) in the backhaul segment. Three well-know examples of RANs are shown in Figure 2: the UTRAN (for UMTS), E-UTRAN (LTE), and a WiFi hotspot. However, it should be noted that the proposed architecture is generic enough to support other RAN technologies as well, both already existing ones (such as WiMAX), or future ones.

In the SDWN architecture, radio access networks are enhanced with programmability (as introduced in more detail below), supporting multiple functionality levels to allow for incremental deployments. The core transport is composed of programmable L2 switches and L3 routers, allowing set-up of unicast and multicast forwarding at the flow level (as supported for example by OpenFlow). Multiple (virtual) operators might share part of the radio, backhaul and transport core network, which requires the interconnection of the core control plane entities - in charge of functions such as authentication, authorization, charging, subscriber management, mobility management, QoS provisioning or connection to external services/networks - with the programmable network.

Two different models can be adopted to implement an SDWN architecture: "evolutionary" and "clean slate." The evolutionary model allows for incremental deployment in existing networks: legacy control plane entities from the operators can connect to the transport core network without modifying the existing interfaces. In this model, the SDN controller implements standardized interfaces to support the internetworking with existing legacy entities, even if they run on a virtualized environment (what is known as virtual EPC, vEPC).

In the clean slate model, the control plane functions are directly programmed on the SDN controller or on top of it as applications, using a software API between the virtual operators and the SDN controller. While this approach does not allow for an easy incremental deployment, it brings from day one all the advantages of programmable network architectures. For example, the deployment of new network functions and services is much easier and faster, as it can be directly implemented on the controller and does not need to impact on multiple interfaces and equipment from different vendors. We can just take the simple, but very representative, example of IPv6 support on a mobile network. With the clean slate approach, adding IPv6 support would just require additional code on the SDN controller, as compared to defining new interfaces, procedures on the different control and user plane entities, which require software/firmware updates (if not even replacing some hardware).
The brain of the architecture, the SDN controller, is connected to each programmable entity. Note that the SDN controller is a logical entity, which might also be decentralized into different physical boxes to improve scalability and performance, though this is currently the subject of extensive research [11].

In order to allow third parties (such as service and application providers) to influence/control the behavior of the network, an API is enabled. This API effectively enables external players to get access to the network resources, similar to what an OS does with the access of applications to computational resources and peripherals. The API offered by the SDN controller supports different access levels to the external parties, so personalization can vary on different dimensions: per application, per user, per (virtual) operator, per access network, or a combination of them.

\section{B. Key Interfaces}

We now focus on the description of the different interfaces (see Figure 3):

- A northbound interface to the (virtual) operators sharing the same physical set of network resources allowing them to dynamically change the share of resources, for example to adapt to network load or to the number and profile of users attached to the physical shared network at any given moment of time. This interface should be able to implement richer SLAs as compared to the ones available nowadays, as a more dynamic and almost real-time reconfiguration of the network would be possible. Each (virtual) operator should have access to an abstracted view of its assigned resources, so they can program that "virtual" network as a physical one.

- A northbound interface to the external parties (service and application providers) authorized to influence the network behavior. This interface should be properly secured, granting access with different granularities and permissions. The interface should be powerful enough to allow an application provider to influence how its traffic is handled, even taking into consideration the virtual operator its users are getting access from. Note that this is possible because of the centralization achieved by the use of the SDN approach, though this may introduce scalability issues (e.g., up to per flow signaling, need for frequent network monitoring, etc) that need to be taken into account.

- A southbound interface to the physical user-plane network entities in the core transport backbone. This interface is used by the SDN controller to implement the different behavior policies according to the requests from the external parties, the virtual operators associated to the different users attached to the network, and the network conditions. Given the logical centralization provided by SDN, close to maximum utilization of the capacity of the network links can be achieved. This interface also allows for effective sharing of a common backbone and 
backhaul network by different operators, which may even connect to the Internet via different gateways.

- A southbound interface to the physical user-plane entities in the RAN. This interface allows for effective virtualization of the access network, therefore sharing the same physical resources among different operators. Besides, this interface should allow programming the wireless access technologies to provide the expected behavior, depending on the specific needs and characteristics of the mobile terminal, the requests from the external providers and the different SLAs that the virtual operators may have in place with their users.

- A southbound interface with the mobile node. This interface provides the network with certain programmability capabilities on the mobile node. This can be used for example to improve the mobility experience, by better exploiting the simultaneous use of available wireless access networks, e.g., helping in access network and interface selection.

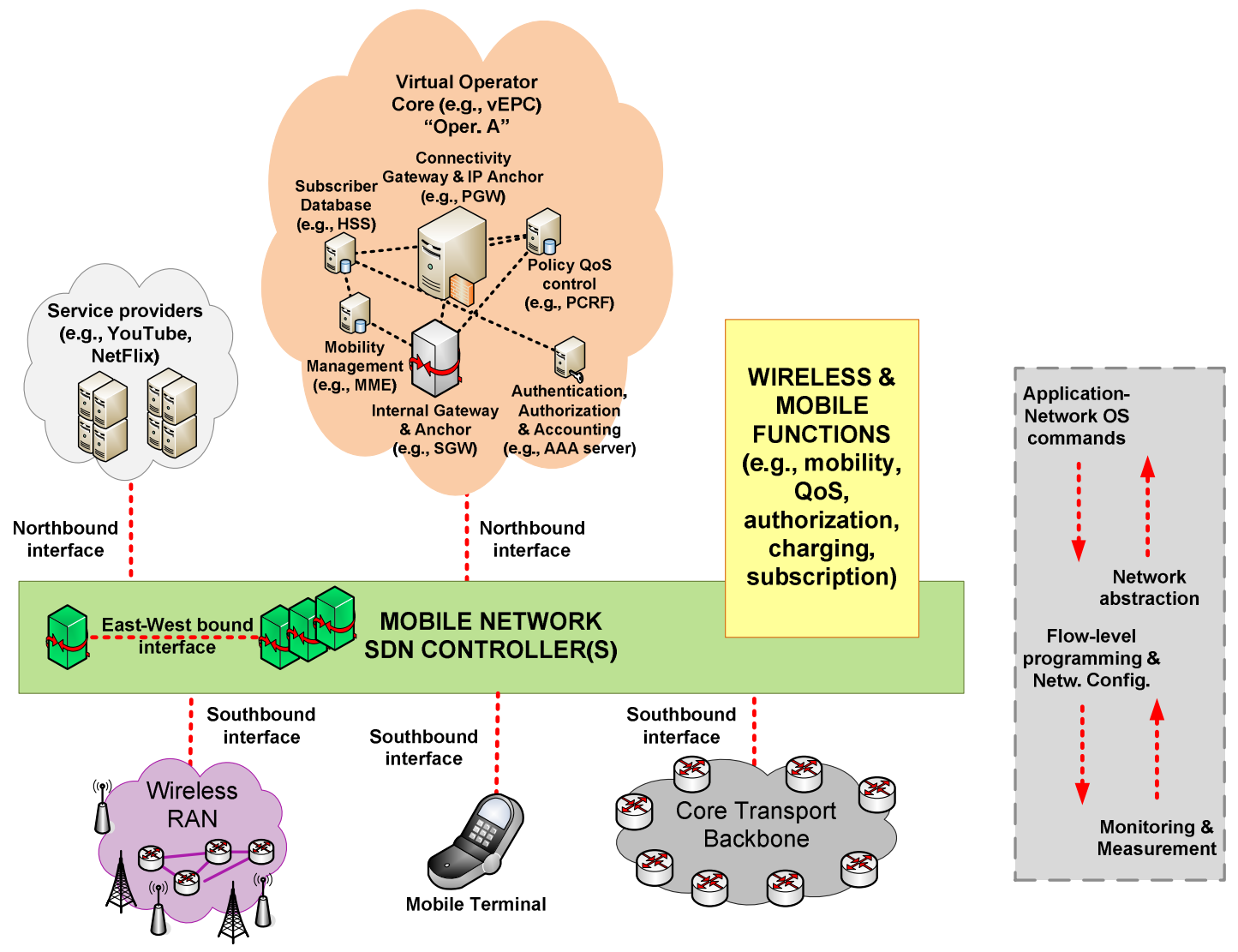

Figure 3. SDN-based mobile network interface architecture

A proper implementation of the above interfaces, together with the required intelligence on the SDN controller will provide new functions/flexibility, not available on today's architectures. We next describe some examples.

The logical centralization of SDWN offers the ability to change the forwarding of user data traffic in the access (radio and backhaul) and core transport networks, which can be used to provide functionalities such as mobility management and QoS provisioning.

The programmable configuration of the RAN (including the backhaul), e.g., MAC and radio access parameters, allows for best dynamic use of available resources, considering current load, users' distribution and network sharing among virtual operators. Examples of this are: configuration of the Wireless Multimedia Extensions for $\mathrm{WiFi}$, configuration of radio bearers for 3GPP accesses, configuration of IEEE 802.11aa behavior for multicast transmission over $\mathrm{WiFi}$, management of optical and microwave parameters in backhaul links, etc. Overall, this programmability can be used to help meeting both global network-wide goals (in terms of efficiency), as well as particular targets on a per virtual operator and service provider basis (e.g., differentiation on a per application/mobile node).

The joint dynamic configuration of the RAN and transport core network allows performing global network updates to better adapt to current conditions. Examples of these updates are using different unicast/multicast distribution schema within the network in order to better meet specific QoS requirements. But this can also be used to offer dynamic adaptation of the traffic at transport and application level, for 
example, NAT or IPv6/IPv4 transition functionality can be dynamically enabled and moved within the network. Even more, the forwarding path of selected multimedia flows can be updated, so they traverse a middleware box capable of adapting the content to the network and client conditions (by re-encoding the multimedia stream, or selectively dropping some packets, for example).
Extending the programmability to the mobile nodes enables very interesting enhancements on the users' experience. Handover management can be made much more efficient, as network and client side would be in tight coordination, which eases tasks such as cellular offloading and faster handovers. Network discovery can also be simplified, as the network can enforce its policies more easily and almost in real time.

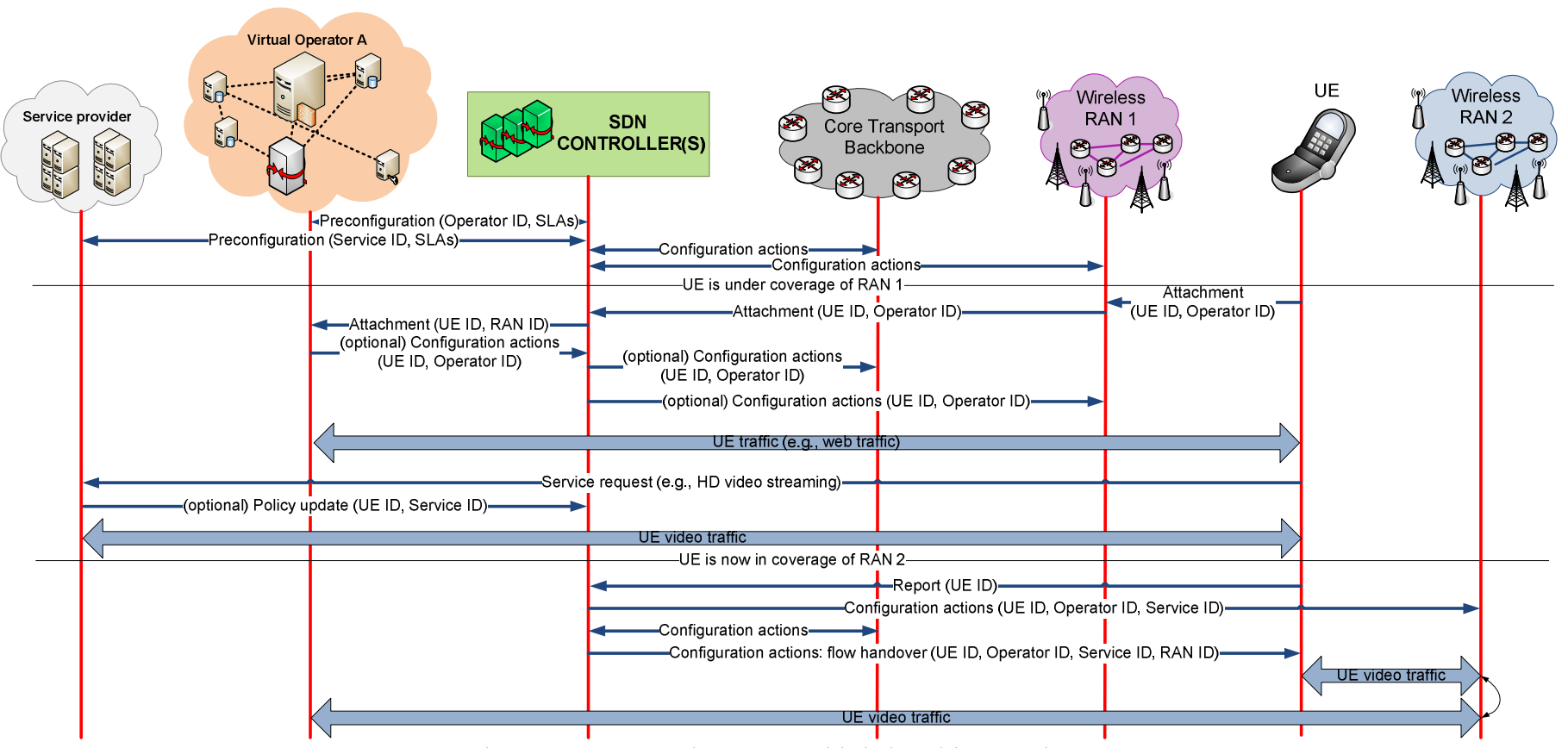

Figure 4. Case study: SDWN high-level interactions

\section{Case study}

In order to show how the proposed SDWN framework works, we next make use of an example case study, describing the high level interactions between the main SDWN components (see Figure 4). We not only explain how to make use of the defined interfaces - highlighting the opportunities enabled by the use of SDN in a wireless mobile network - but also identify some of the challenges posed by the adoption of such an architecture.

In our case study - which is meant to serve just as an example, and therefore it does not capture all the possible interactions a virtual operator A operates using the resources of a core transport backbone and different radio access networks. Using the defined northbound interface with the SDN controller(s), Virtual Operator A is capable of pre-configuring the physical network resources, so it appears as a valid operator in the area covered by wireless access networks. Similarly, a service provider can also pre-configure the network in order to provide a certain default treatment to its traffic (e.g., provide low end-to-end latency). Note that in both cases, the use of the interfaces provided to configure/influence the behavior of the network requires proper service level agreements (SLAs) to be in place.

In this scenario, if a user equipment (UE) falls within the coverage of the Wireless RAN 1 and attaches to the network, this event can be reported to the SDN controller(s), using the existing southbound interface. This attachment event is also notified to the Virtual Operator A, which can optionally trigger some specific configuration actions on the network for that particular UE. These additional configurations can affect both the transport core and the wireless access networks, for example to offer a prioritized wireless access, by configuring the MAC service provided to the UE.

If the UE requests a service for which the network has an agreement in place (video in this example), its traffic is provided with a differentiated service. The network configuration required to do so can be already in place, or can be triggered on the service provider by the SDN controller(s). Both approaches are possible (pre-configuration of the traffic forwarding and on-demand dynamic configuration), allowing for different service models (e.g., gold users are provisioned on demand, while regular users are provided with a default service). Note that scalability might be a concern if preconfigured policies are not used, and the traffic from all the users is treated on-demand. This is one of the main challenges to be addressed in SDN architectures.

If the UE gets into coverage of another wireless access network, also managed by the same SDN controller(s), and with the required SLAs in place, this event is received by the network, which can then evaluate to selectively move some flows to the new access, in order to improve this particular 
UE's QoE, the overall utilization of the network, or both. In this example, video traffic is moved to the Wireless RAN 2, by exploiting the existing SDN interfaces with both the infrastructure network entities and the UE.

\section{StandardizATION: STATUS AND FUtURE NEEDS}

\section{A. Current efforts}

Regarding standardization efforts in the area of SDN, the most relevant Standards Developing Organization (SDO) is the Open Networking Foundation (ONF) $)^{4}$, a member-driven standards organization aiming to promote and adopt Software Defined Networking through open standards development. ONF is the home of the well-known OpenFlow standard and OpenFlow Switch Specification [5], defining the protocol used for the communication between the OpenFlow controller and switches. In addition to these core standards, the ONF also publishes a testing specification to guide the conformance of the OpenFlow switches [12]. ONF is structured in several working groups (WGs), which address topics that range from extensibility (adding new features) to migration (for existing networks to adapt to the OpenFlow standard). Recently, a Wireless and Mobile Working Group has been established to address the specific requirements of mobile networks. The charter of the group ${ }^{5}$ lists a number of identified use cases ranging from mobile backhaul to mobile core issues. OpenFlow extensions can be expected in near future to cover the gap in existing specifications.

At the $\mathrm{IETF}^{6}$, the SDN trend is impacting several WGs. The most representative SDN-related WGs are the following: the Forwarding and Control Element Separation (FORCES), which is tackling the separation between the control plane and data plane; the Network Virtualization Overlays (NVO3), focusing on the data center overlay problem space and architecture; and finally, the Interface to the Routing System (I2RS), which focuses on providing a real-time interface into the IP routing system.

In addition, the IRTF has created the Software-Defined Networking Research Group ( $\mathrm{SDNRG}^{7}$ ), which is analyzing the approaches that can be used both in the near term and in the future. Finally, there are some other WGs at the IETF that can bring useful knowledge into SDN standardization, such as ALTO, PCE, NETCONF, NETMOD and DMM WGs.

The IEEE 802 LAN/MAN Standards Committee has also started SDN-related activities. Although there is currently no WG focused specifically on SDN in IEEE 802, there are ongoing discussions on how to introduce SDN capabilities on wireless and wired technologies. The most relevant activities are: the Open Mobile Network Interface for Omni-Range Area Networks (OmniRAN) Study Group, defining an access network specification based on IEEE 802 technologies (e.g., $802.11,802.1 \mathrm{X}$, etc.) that includes a network architecture, recommended communication protocols, and link-specific

\footnotetext{
${ }^{4}$ https://www.opennetworking.org/

${ }^{5}$ https://www.opennetworking.org/images/stories/downloads/working-

groups/charter-wireless-mobile.pdf

${ }^{6} \mathrm{http}: / /$ www.ietf.org/

${ }^{7}$ http://irtf.org/sdnrg
}

parameters usage, which has specifically discussed the SDN capabilities that are needed in the specific IEEE 802 technologies [13]; ongoing discussion within the IEEE 802.16 $\mathrm{WG}$, to introduce the bridging capability directly within the wireless stack in such a way that SDN functionalities can be applied to the wireless connections [14]; and the IEEE $802.11 \mathrm{ak}^{8}$ and $802.1 \mathrm{Qbz}^{9}$ specifications, which are very relevant as they specify how 802.11 access points (APs) interconnect with wired bridged technologies in a simpler (and more SDN-friendly) manner.

The 3GPP is not currently addressing directly how to introduce SDN concepts on their specifications although SDNbased approaches are being investigated as a possible solution to some current challenges. For example, the concept of reconfigurable backhaul is used for RAN sharing. The sharing of the radio access network by different operators requires the traffic to be routed to the correct network, depending on the operator policies. In addition to this functionality, SDN-related concepts are being discussed as a technology that can be applied to Self-Organizing Networks (SON) [15]. SON networks provide mechanisms for self-optimization, selfhealing and self-configuration, applying concepts such as the programmable control of the network, which are shared by SDN-based approaches.

Other SDOs such as ETSI or ITU-T are also considering approaches similar to SDN for defining their architectures for Future Networks. This is the case of ITU-T SG $13^{10}$ (Future networks including cloud computing, mobile and nextgeneration networks), which has included in its work programme several topics related with SDN networks, as mechanisms for realizing the Network Intelligence Capability Enhancement (NICE), developed at the ITU-T. In the case of ETSI, the Autonomic network engineering for the selfmanaging Future Internet (AFI) Industry Specification Groups (ISG) is working on solutions for autonomic (self-) management and control of the network resources in mobile networks.

Additionally, ETSI has recently (October 2013) delivered several specifications on Network Function Virtualization ${ }^{11}$ (NFV). Although NFV and SDN concepts are mutually beneficial but not dependent on each other, both proposals share the idea of providing a set of functions enabling the programing of the network functions.

\section{B. Future Needs}

Standardization efforts represent one of the key building blocks of the telecommunication industry. A standard answers to the need for communication among devices of different manufacturers. Hence the focus of a standard is placed on protocol definition, message formats and corresponding behaviors, leaving the specific implementations to the choice of the manufacturer. For example, OpenFlow, one of the most relevant SDN tools available, uses a central controller and a

\footnotetext{
${ }^{8} \mathrm{http}: / /$ www.ieee802.org/11/Reports/tgak_update.htm

${ }^{9} \mathrm{http}: / /$ www.ieee802.org/1/pages/802.1bz.html

${ }^{10} \mathrm{http}: / /$ www.itu.int/en/ITU-T/studygroups/2013-2016/13/Pages/default.aspx

${ }^{11} \mathrm{http}: / /$ www.etsi.org/technologies-clusters/technologies/nfv
} 
set of interfaces, namely a Northbound interface to communicate with control applications and a Southbound interface to communicate with the actual network hardware. Through these two interfaces, applications can be developed to use network functions and interact with the deployed hardware. Hence, it is of vital importance to standardize these two interfaces in order to be able to communicate and interact with hardware provided by different vendors.

Currently, different SDOs are working on very related and entangled issues although each one is focused on a different point of view. As such, the current panorama of standardization activities is formed by a number of noncollaborative activities, several of them trying to solve the same issues in a slightly different way. SDN relies on APIs and standardized Service Access Points (SAPs), to be able to control the behavior of physical network elements. As such, standardization plays a crucial role for this purpose, since without a clear definition of these interfaces, SDN cannot become a reality. In order to organize and focus the work performed by the different bodies addressing the important labor of standardizing SDN related technologies, it is desirable to create a common view of use cases, network services and functionality among all SDOs, which can be further used to create a real momentum and push forward the next generation of SDN concepts.

Table 1: The case for SDN in Mobile Networks

\begin{tabular}{|l|l|}
\hline \multicolumn{1}{|c|}{ Key Benefits } & \multicolumn{1}{c|}{ Key Challenges } \\
\hline $\begin{array}{l}\text { Easier deployment of new } \\
\text { services }\end{array}$ & Specification of the interfaces \\
\hline $\begin{array}{l}\text { Reduced management and } \\
\text { operational costs of } \\
\text { heterogeneous technologies }\end{array}$ & $\begin{array}{l}\text { Need to integrate scheduled- } \\
\text { based and contention-based } \\
\text { systems }\end{array}$ \\
\hline $\begin{array}{l}\text { Efficient operation of multi- } \\
\text { vendor infrastructures }\end{array}$ & $\begin{array}{l}\text { Harmonization of the } \\
\text { standardization efforts }\end{array}$ \\
\hline $\begin{array}{l}\text { Increased accountability and } \\
\text { service differentiation }\end{array}$ & $\begin{array}{l}\text { Verifiable security and privacy } \\
\text { architecture }\end{array}$ \\
\hline $\begin{array}{l}\text { Continuous and transparent } \\
\text { enhancement of network } \\
\text { operation }\end{array}$ & $\begin{array}{l}\text { Operation and management of } \\
\text { wireless networks is more } \\
\text { complex }\end{array}$ \\
\hline
\end{tabular}

\section{CONCLUSIONS}

In this article we have identified the opportunities that software defined networking can bring to wireless and mobile networks. We propose a high-level architecture leveraging on the advantages of the logical centralization provided by SDN. We have first defined the main functions that should be supported by a mobile SDN architecture, and then specified the required interfaces and describe some of the interactions that would be needed to enable new and/or richer use cases. A summary of the key benefits and challenges for SDN in mobile networks is provided in Table 1.

Last, but not least, we have reviewed ongoing standardization efforts around SDN topics, identifying the future needs to ensure a successful practical deployment of SDN mechanisms in the wireless arena.

\section{ACKNOWLEDGEMENTS}

The research leading to these results has been partly funded by the European Community's Seventh Framework Program FP7/2007-2013 under grant agreement $n^{\circ} 317941$ - project iJOIN, http://www.ict-ijoin.eu/.

\section{REFERENCES}

[1] Y.-J. Chang et al., "Scalable and Elastic Telecommuncation Services in the Cloud”, Bell Labs Technical Journal, vol. 17, no. 2, pp. 81-96, 2012.

[2] "Network Functions Virtualisation - An Introduction, , Benefits, Enablers, Challenges, and Call for Action", white paper available at http://portal.etsi.org/NFV/NFV_White_Paper.pdf, 2012.

[3] S. Jain et al., "B4: experience with a globallydeployed software defined WAN," in Proceedings of the ACM SIGCOMM 2013 conference on SIGCOMM, ser. SIGCOMM '13. New York, NY, USA: ACM, 2013, pp. 3-14.

[4] Open Network Foundation, "Software-Defined Networking: The New Norm for Networks", 2012.

[5] Open Network Foundation, OpenFlow Switch Specification, v1.4.0. October 14, 2013.

[6] P. Calhoun, M. Montemurro, D. Stanley "Control And Provisioning of Wireless Access Points (CAPWAP) Protocol Specification”, RFC 5415, March 2009.

[7] G. Bianchi et al., "MAClets: active MAC protocols over hard-coded devices," in Proceedings of the 8th international conference on Emerging networking experiments and technologies (CoNEXT '12), ACM, New York, NY, USA, pp. 229-240, 2012.

[8] K. Pentikousis et al., "Mobileflow: Toward software-defined mobile networks," Communications Magazine, IEEE, vol.51, no.7, pp.44,53, July 2013.

[9] NGMN, "Backhaul Evolution - Integrated QoS Management".

[10] A. de la Oliva et al., "IP flow mobility: smart traffic offload for future wireless networks," Communications Magazine, IEEE, vol.49, no.10, pp. 124,132, Oct. 2011.

[11] A. Dixit et al. "Towards an elastic distributed SDN controller," in Proceedings of the second ACM SIGCOMM workshop on Hot topics in software defined networking (HotSDN '13). ACM, New York, NY, USA, 7-12.

[12] Open Network Foundation, "Conformance Test Specification for OpenFlow Switch Specification”, v1.0.1, June 13, 2013.

[13] R. Marks, A. de la Oliva and J.C. Zuñiga, "Proposed OmniRAN SDN Use Case for External Communication", August 2013.

[14] IEEE P802.16r, "View of Connection-Oriented Software-Defined Networking for Wireless Backhaul of Small Cells", July 2013.

[15] 3GPP, TS 32.500, Telecommunication Management; "Self-Organizing Networks (SON); Concepts and requirements", December 2011.

\section{BIOGRAPHIES}

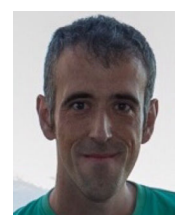

Carlos J. Bernardos received a Telecommunication Engineering degree in 2003, and a $\mathrm{PhD}$ in Telematics in 2006, both from Universidad Carlos III de Madrid (UC3M), where he worked as a research and teaching assistant from 2003 to 2008 and, since then, has worked as an Associate Professor. His current work focuses on mobility in heterogeneous wireless networks. He has published over 50 scientific papers in international journals and conferences, and he is an active contributor to the IETF. He has served as guest editor of IEEE Network.

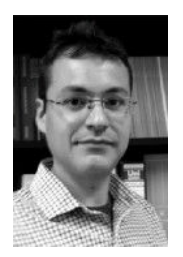

Antonio de la Oliva received a Telecommunication Engineering degree in 2004, and a PhD in Telematics in 2008 (receiving a national award ex-aequo for the best $\mathrm{PhD}$. thesis in IPTV services), both from the University Carlos III of Madrid (UC3M), where he worked as a research and teaching assistant from 2005 to 2008 and, since then, has worked as an Visiting Professor. His research is focused to mobility in heterogeneous networks and wireless systems. He has 
published over 30 scientific papers in prestigious international journals and conferences, and he is also an active contributor and voting member of the IEEE 802.21 where he has served as Vice-chair of IEEE 802.21b and Technical Editor of IEEE 802.21d. Currently he is involved in the FP7 CROWD project.

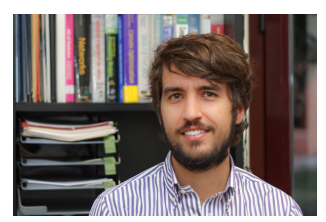

Pablo Serrano got his Telecommunication Engineering degree and his $\mathrm{PhD}$ from the Universidad Carlos III de Madrid (UC3M) in 2002 and 2006, respectively. He has been with the Telematics Department of UC3M since 2002, where he currently holds the position of Associate Professor. He was a visiting researcher at the Computer Network Research Group at Univ. of Massachusetts Amherst in 2007, and at Telefonica Research Center in Barcelona in 2013. He has over 50 scientific papers in peer-reviewed international journal and conferences. He serves on the Editorial Board of IEEE Communications Letters, and has been a guest editor for Computer Networks.

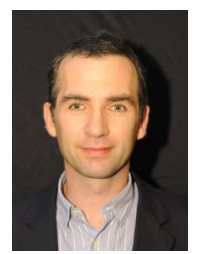

Albert Banchs received his degree in telecommunications engineering from the Polytechnic University of Catalonia in 1997, and his Ph.D. degree from the same university in 2002. He received a national award for the best Ph.D. thesis on broadband networks. He was a visiting researcher at ICSI, Berkeley, in 1997, worked for Telefonica I+D, Spain, in 1998, and for NEC Europe Ltd., Germany, from 1998 to 2003. He has been with the University Carlos III of Madrid since 2003. Since 2009, he also has a double affiliation as Deputy Director of the IMDEA Networks institute.

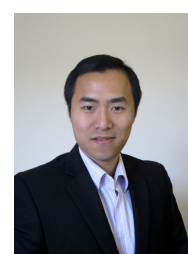

Hao Jin is a senior engineer at InterDigital Communications, Inc., where he is currently working on the research and development of SDN and network virtualization for wireless and mobile networks. He earned his B.S. degree in Electrical Engineering from Nanjing University, China in 2006, and his Ph.D. degree in Electrical Engineering from Florida International University in 2012. His research interests include SDN, network virtualization, next generation wireless networks, and data center networking.

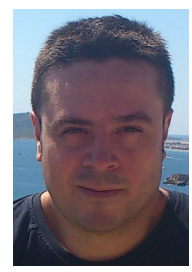

Luis M. Contreras completed a six-year Telecom Engineer degree at the Universidad Politécnica of Madrid (1997), and holds an M. Sc. on Telematics from UC3M (2010). In 1997 he joined Alcatel in Spain taking several positions in both wireless and fixed network fields. In 2006 he joined the Network Planning department of Orange in Spain (France Télécom group) taking responsibilities on the IP backbone and mobile packet core planning. Between 2002 and 2010 he was also adjunct lecturer at the Telematics department of UC3M, where he is currently a Ph.D. student. Since August 2011 he is part of Telefónica I+D / Telefónica Global CTO, working on SDN, transport networks and their interaction with cloud and distributed services. $\mathrm{He}$ is active contributor to IETF and ONF.

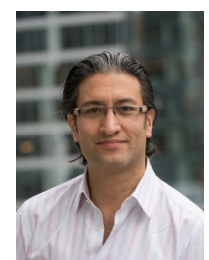

Juan Carlos Zúñiga received his engineering degree from the UNAM, Mexico, and his MSc and DIC from the Imperial College London, UK. He is a Principal Engineer at InterDigital, currently leading the standardization activities in the areas of heterogeneous networks, software defined networking, distributed content management and Internet privacy. He has actively contributed and held leadership roles in different standards fora, such as IEEE 802, IETF, and 3GPP. He has been with InterDigital since 2001. Previously he worked with Harris Communications in Canada, Nortel Networks in the UK, and $\mathrm{Kb} / \mathrm{Tel}$ in Mexico. He is an inventor of more than 30 granted U.S. patents and 70 published applications. 